12

\title{
Синтез электронно-оптических систем с компрессией ленточного пучка для ламп бегущей волны терагерцевого диапазона
}

\author{
(C) А.А. Бурцев, ${ }^{1,3}$ А.В. Данилушкин, ${ }^{2}$ Н.И. Синицын ${ }^{3}$ \\ ${ }^{1}$ Акционерное общество «Научно-производственное предприятие «Алмаз», \\ 410033 Саратов, Россия \\ ${ }^{2}$ Саратовский государственный технический университет им. Гагарина Ю.А., \\ 410054 Саратов, Россия \\ ${ }^{3}$ Саратовский фрилиал Института радиотехники и электроники им. В.А. Котельникова РАН, \\ 410019 Саратов, Россия \\ e-mail: antbourtsew@gmail.com
}

(Поступило в Редакцию 8 февраля 2018 г. В окончательной редакции 25 мая 2018 г.)

\begin{abstract}
Представлены результаты синтеза электронно-оптических систем формирования сходящегося ленточного электронного пучка с компрессией 15 и 20 , сечением $0.05 \times 2 \mathrm{~mm}$ и с плотностью тока $100 \mathrm{~A} / \mathrm{cm}^{2}$ при полной магнитной экранировке автоэмиссионного катода. Проведен анализ деформации в пролетном канале замедляющей структуры низкопервеансного потока в магнитном поле на основе компьютерного трехмерного моделирования электронно-оптической системы с ленточным электронным пучком.
\end{abstract}

DOI: $10.21883 / J T F .2018 .12 .46797 .54-18$

Мощные миниатюрные электровакуумные источники когерентного терагерцевого излучения диапазона частот 0.2-0.3 THz могут быть реализованы на основе электровакуумных приборов таких, как лампы бегущей волны (ЛБВ), где используются ленточные электронные пучки с высокой плотностью тока [1,2]. Одной из основных проблем при создании вакуумных приборов терагерцевого диапазона является необходимость использования в узких пролетных каналах замедляющих систем электронные пучки с уровнем плотности тока до $500 \mathrm{~A} / \mathrm{cm}^{2}$, что является трудноосуществимым из-за ограниченной эмиссии современных катодов в прямоточной оптике. Поэтому перспективны те электронно-оптические системы (ЭОС), в которых используются сходящиеся ленточные пучки, позволяющие получить достаточно большие плотности тока при меньшей токовой нагрузке на катод и с меньшим значением магнитного поля.

Существуют несколько вариантов реализации ЭОС формирования сходящегося ленточного пучка. В одном из них катод пронизывается магнитным полем, при этом обеспечивается лучшая структура электронного пучка в пролетном канале за счет некоторого подавления тепловых скоростей электронов на катоде и меньшей зависимости от возмущающих факторов таких, как, например, несоосность элементов ЭОС. Второй вариант - полностью магнито-экранированная пушка. В этом случае величина рабочего магнитного поля может быть меньше при тех же размерах пучка и первеансе. Пучок становится менее чувствительным к влиянию переходной области магнитного поля и степени экранировки катода. Поскольку уровни фокусирующих магнитных полей минимальны, в настоящей работе для проектирования ЭОС, формирующих ленточные пучки, рассматривается вариант с магнито-экранированным катодом.
При разработке ЭОС для приборов О-типа терагерцевого диапазона дополнительно возникает задача фокусировки и согласованного ввода ленточного пучка в магнитное поле. Трудность токопрохождения ленточного пучка в пролетном канале из-за его деформации предполагает анализ поведения электронов на краях пучка.

В настоящей работе используются модель ламинарного пучка и криволинейная система координат (рис. 1), при этом ширина электронного пучка больше его толщины. Это позволяет предположить, что распределение плотности тока и заряд в пучке не зависят от поперечной по ширине пучка координаты $q_{3}$. Уравнение внутренней задачи синтеза, согласно [3], имеет вид

$$
\left(u^{\prime} \varphi\right)^{\prime}+2 \varphi^{\prime \prime} u+\gamma^{2}\left(h^{2} \varphi-h h_{c} \varphi_{c}\right)=\frac{i}{\sqrt{u}},
$$

где $\varphi-$ функция, описывающая границу пучка; $u, h-$ нормированные осевые потенциал и магнитное поле; $h_{c}$ и $\varphi_{c}-$ значение функций $h$ и $\varphi$ на катоде, $i=0.0952 \frac{p_{\mu}}{\mu \mu_{1}}, \quad \mu=\Phi_{0} / l, \quad \mu_{1}=s / l ; p_{\mu}$ - микропервеанс; $\Phi_{0}, l$ - нормировочные величины поперечных и продольных размеров ЭОС, $s$ - ширина пучка, $\gamma^{2}=\eta B^{2} l^{2} / U_{0} \quad\left(B\right.$ и $U_{0}-$ нормировочные величины магнитного поля и потенциала).

Уравнение внутренней задачи (1) содержит три искомые функции $\varphi(x), u(x), h(x)$ и для его решения необходимо задаваться двумя из них. В области, где осевой потенциал $u=1$ и осевое магнитное поле $h_{0}$, причем на катоде магнитное поле отсутствует $\left(h_{c}=0\right)$, из уравнения (1) можно записать: $\gamma^{2} h_{0}^{2} \varphi_{0}=i$, откуда можно выразить $\gamma$. Тогда уравнение (1) запишется в следующем виде:

$$
\left(u^{\prime} \varphi\right)^{\prime}+2 \varphi^{\prime \prime} u+i h^{2} \frac{\varphi}{\varphi_{0}}=\frac{i}{\sqrt{u}} .
$$




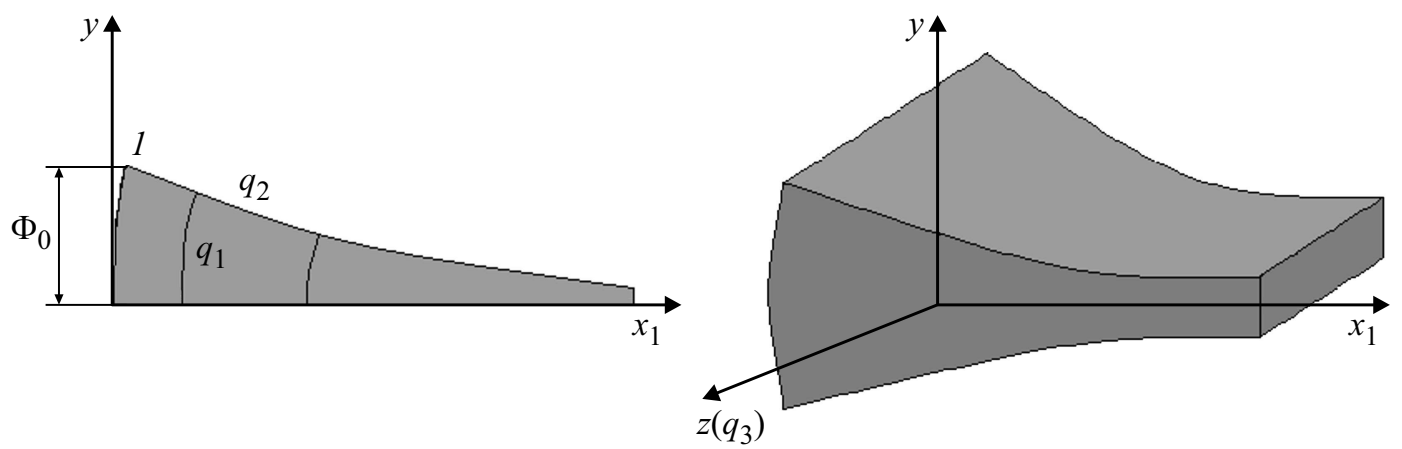

Рис. 1. Конфигурация ленточного пучка и системы координат.

Распределение магнитного поля в магнито-экранированной пушке в области нарастания потенциала до $u=1$ примем в следующем виде [4,5]:

$$
h=\sin ^{2}\left(x_{1}-x_{m}\right),
$$

где $x_{1}-$ продольная координата, $x_{m}-$ координата начала нарастания магнитного поля в ЭОС.

В области, где осевой потенциал $u=1$, из уравнения (2) получим выражение для распределения магнитного поля $h$ :

$$
h=\sqrt{\frac{\varphi_{0}}{\varphi}-2 \frac{\varphi^{\prime \prime}}{i} \frac{\varphi_{0}}{\varphi}} .
$$

Перспективными ЭОС формирования ленточных электронных пучков источников излучения О-типа терагерцевого диапазона являются системы, использующие эффект автоэлектронной эмиссии. Моделирование таких систем может быть проведено методом синтеза с использованием модели ЭОС с гладкой поверхностью катода и наличием напряженности электрического поля на его поверхности, которая за счет усиления электрического поля в автоэмиттерах в катоде достигает величины, необходимой для автоэмиссии.

В настоящей работе распределение потенциала вблизи поверхности катода определяется уравнением

$$
\left(u^{\prime} \varphi\right)^{\prime}+2 \varphi^{\prime \prime} u=\frac{i}{\sqrt{u}}
$$

Из уравнения (5), как это показано в работе [6], можно аналитически получить выражение для напряженности электрического поля

$$
\begin{aligned}
& u^{\prime}(x)= \\
& =\sqrt{2.7 \sqrt{x u_{c}^{\prime}} i \varphi_{c}^{\prime} x+\left[2 i \sqrt{u}\left(1-\varphi_{c}^{\prime} x\right)+\left(u_{c}^{\prime}\right)^{2}\left(1-\varphi_{c}^{\prime} x\right)^{2}\right]},
\end{aligned}
$$

при этом функция $\varphi$ вблизи автоэмиссионного катода задается в виде $\varphi=\varphi_{c}^{\prime} x+1$. Далее в пушке распределение потенциала задается в виде

$$
u(x)=\sum_{0}^{6} a_{n}\left(x-x_{1}\right)^{n},
$$

где первые три коэффициента находятся из условий сшивания потенциала, его первой и второй производной, а остальные - из условий $u\left(x_{2}\right)=1, u^{\prime}\left(x_{2}\right)=u^{\prime \prime}\left(x_{2}\right)=0$; функция $\varphi$ находится из решения уравнения внутренней задачи синтеза (2).

Нахождение конфигурации фокусирующего электрода и анода пушки следует из решения уравнения внешней задачи синтеза, имеющей, согласно [7], вид

$$
V\left(x, q_{2}\right)=u+\frac{\mu^{2} i \varphi}{2 \sqrt{u}}\left(2 q_{2}-1\right)-\frac{\mu^{2} q_{2}^{2}}{2}\left(\varphi^{2} u^{\prime \prime}+\varphi \varphi^{\prime} u^{\prime}\right) .
$$

Переход от криволинейной системы координат к декартовой осуществляется с помощью уравнения

$$
\frac{d \xi}{d q_{2}}=-\frac{\mu^{2} \varphi(\xi) \varphi^{\prime}}{1+\left(\mu q_{2} \varphi^{\prime}(\xi)\right)^{2}} q_{2}
$$

где $q_{2}-$ поперечная криволинейная координата, $\xi=x / l-$ продольная декартова координата, $y / l=\mu q_{2} \varphi(\xi)$ - поперечная декартова координата. Далее вычисляются нормировочные размеры, осевая длина пушки, линейная компрессия пучка, радиус кривизны катода. Магнитное поле в пушке вдоль нормированной координаты $x_{1}$ определяется по формуле $B=h(x) \sqrt{6.9 \cdot 10^{-7}\left(p_{\mu} / s d\right) U}$.

На рис. 2 представлено моделирование ЭОС с автоэмиссионным катодом и ленточным пучком $0.05 \times 2 \mathrm{~mm}$ с линейной компрессией 15 с плоским автокатодом и с компрессией 20 с цилиндрическим автокатодом, имеющим радиус кривизны $6.2 \mathrm{~mm}$. Плотность тока в пучке $100 \mathrm{~A} / \mathrm{cm}^{2}$.

Найденное по формуле $E_{c}=u_{c}^{\prime} U_{0} / l$ значение средней напряженности электрического поля вблизи катода для рассчитанного варианта ЭОС с цилиндрическим автокатодом $\left(0.2 \cdot 10^{5} \mathrm{~V} / \mathrm{cm}\right)$ может, например, определять действующую напряженность поля для необходимой плотности токоотбора в соответствии с формулой ФаулераНордгейма за счет экспериментально измеренного коэффициента усиления поля микро-наноострийного катода. 
Синтезированная ЭОС была также рассчитана в трехмерной программе численного анализа Lorentz-3EM. В результате были получены сечения ленточного пучка в пролетном канале замедляющей структуры (рис. 3), где наблюдается его деформация при разных расстояниях от катода при транспортировке в магнитном поле порядка 0.6 Т. Среднеквадратичный эмиттанс пучка составил $5.3 \cdot 10^{-8} \pi \cdot \mathrm{m} \cdot \mathrm{rad}$. Транспортировка ленточного пучка с коэффициентом заполнения в канале 0.5 дает стопроцентное токопрохождение на длине до $45 \mathrm{~mm}$.

В результате проведенного моделирования была спроектирована ЭОС с ленточным потоком сечением $0.05 \times 2 \mathrm{~mm} \mathrm{c}$ линейной компрессией 15 и 20 и плотностью тока в пучке $100 \mathrm{~A} / \mathrm{cm}^{2}$. Проведенное в рамках данного исследования моделирование сходящегося ленточного электронного пучка, сформированного с помощью магнито- экранированной электронной пушки, показало возможность получения низкопервеансного ленточного потока с незначительной деформацией формы в поперечном сечении, что важно для применения в микроразмерных электродинамических структурах ЛБВ терагерцевого диапазона, при этом амплитуда магнитного поля была выбрана 0.6 Т. Дальнейшее уменьшение
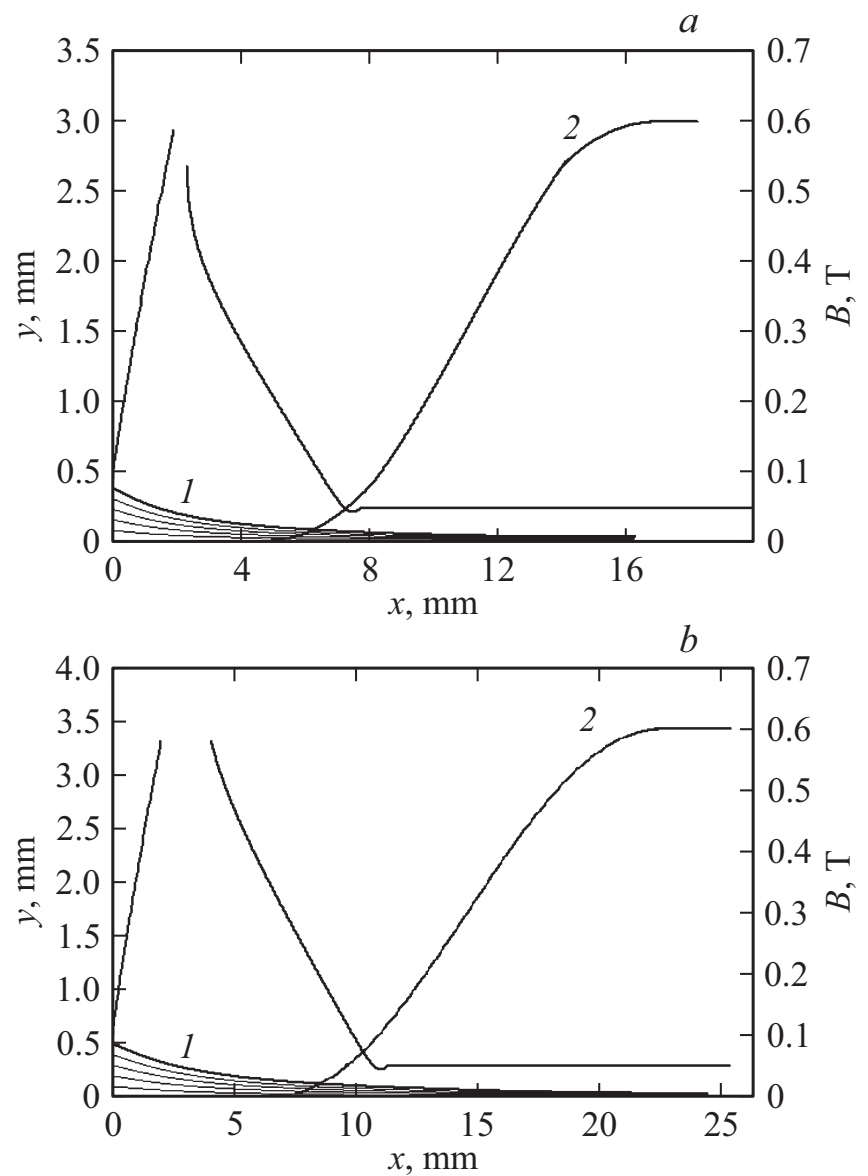

Рис. 2. Результаты моделирования ЭОС: $a-$ с плоским автокатодом с компрессией $15 ; b-$ с цилиндрическим автокатодом с компрессией $20 ; 1-$ траектории электронов $\varphi(x), 2-$ распределение магнитного поля $B(x)$.
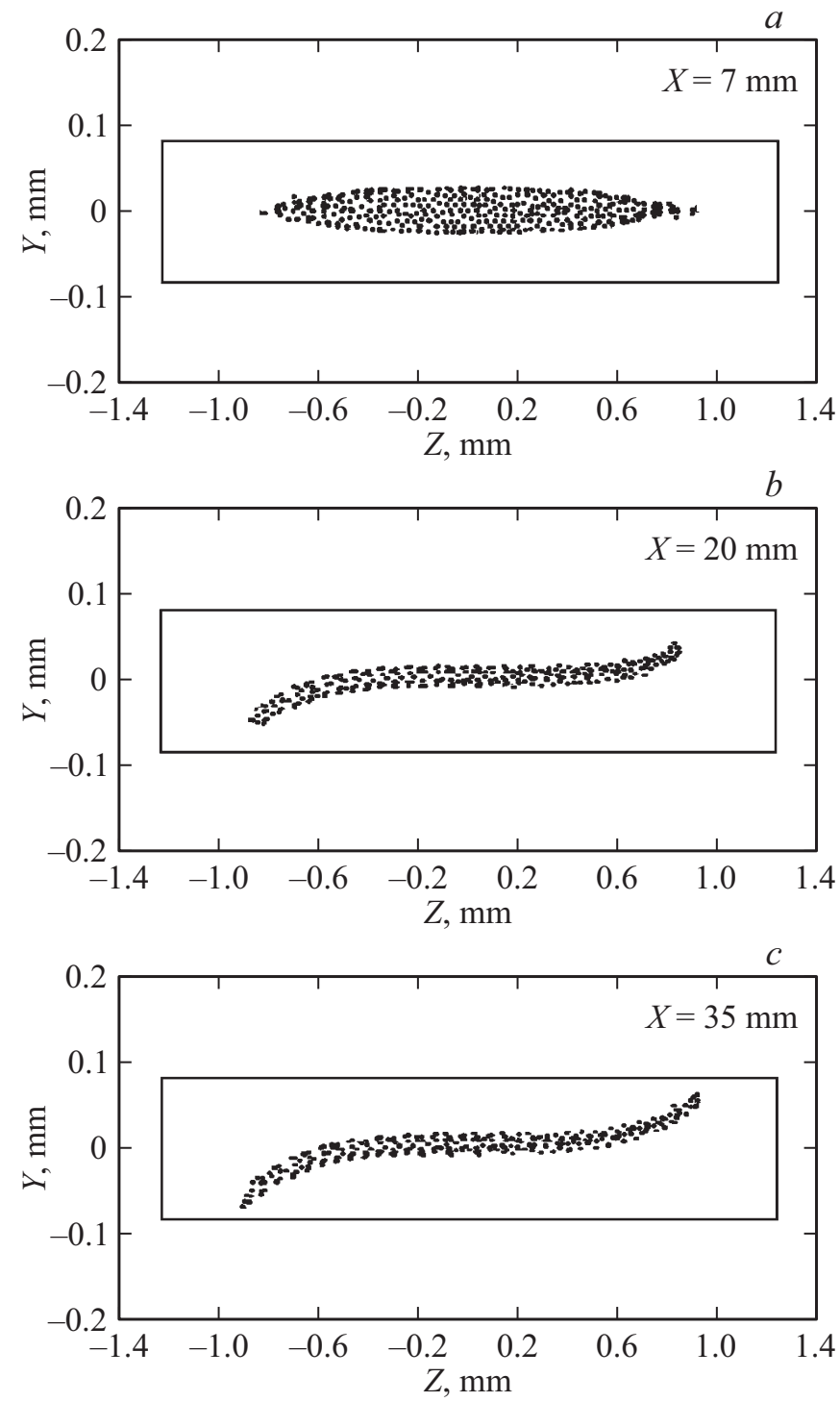

Рис. 3. Сечения ленточного электронного пучка в ЭОС при различных расстояниях от автокатода.

величины угла деформации в данном случае возможно за счет увеличения уровня фокусирующего магнитного поля. Представлено моделирование ЭОС с автоэмиссионным катодом и ленточным пучком $0.05 \times 2 \mathrm{~mm} \mathrm{c}$ компрессией 15 и 20 и током $0.1 \mathrm{~A}$.

Работа выполнена при финансовой поддержке Российского научного фонда в рамках научного проекта № 1712-01160.

\section{Список литературы}

[1] Бурцев А.А., Григорьев Ю.А., Наврочкий И.А., Роговин В.И., Сахаджи Г.В., Шумихин К.В. // Письма в ЖТФ. 2016. Т. 42. Вып. 10. С. 92-98.

[2] Zheng Y., Gamzina D., Popovic B., Luhmann N.C. // IEEE Trans. El. Dev. 2016. Vol. 63. N 11. P. 4466. 
[3] Невский П.В. // Обзоры по электронной технике. Сер. 1. Электроника СВЧ. 1989. № 15. 1483. С. 1.

[4] Алямовский И.В. Электронные пучки и электронные пушки. М.: Советское радио, 1966. 454 с.

[5] Gamayunov Yu.G., Patrusheva E.V. // Int. Conf. on Actual Problems of Electron Devices Engineering. APEDE. 2016. Vol. 1. P. 30.

[6] Gamayunov Y.G., Patrusheva E.V., Grigoriev Y.A., Burtsev A.A., Danilushkin A.V. // IVEC 24-26 April, 2017. London. DOI: 10.1109/IVEC.2017.828954

[7] Овчаров В.T. // Радиотехника и электроника. 1967. Т. 12. № 12. C. 2161. 